\title{
Agradecimento aos Revisores da Revista Brasileira de Cardiologia Invasiva - 2010
}

\footnotetext{
A Revista Brasileira de Cardiologia Invasiva agradece aos membros do Conselho Editorial e aos revisores ad A hoc abaixo listados, responsáveis pela revisão dos trabalhos publicados no ano de 2010, que possibilitaram a publicação de artigos de alto nível científico, dentro do mais absoluto rigor ético:
}

\section{Adriano Caixeta}

Hospital Israelita Albert Einstein

São Paulo, SP

Alexandre do Canto Zago

Hospital de Clínicas de Porto Alegre

Porto Alegre, RS

\author{
Alexandre Schaan de Quadros \\ Instituto de Cardiologia do Rio Grande do Sul/ \\ Fundação Universitária de Cardiologia \\ Porto Alegre, RS \\ André Labrunie \\ Irmandade da Santa Casa de Misericórdia de Marília \\ Marília, SP \\ Hospital do Coração \\ Londrina, PR
}

\section{Antonio Massamitsu Kambara}

Instituto Dante Pazzanese de Cardiologia

Hospital do Coração - Associação do Sanatório Sírio

São Paulo, SP

\section{Ari Mandil \\ Hospital Felício Rocho \\ Belo Horizonte, MG}

\section{Carlos Antonio Mascia Gottschall}

Instituto de Cardiologia do Rio Grande do Sul/

Fundação Universitária de Cardiologia

Porto Alegre, RS

Carlos Augusto Cardoso Pedra

Instituto Dante Pazzanese de Cardiologia

São Paulo, SP

Carlos Augusto Homem de Magalhães Campos Instituto do Coração do Hospital das Clínicas da Faculdade de Medicina da USP

São Paulo, SP

Cesar Rocha Medeiros

Hospital Copa D'Or

Rio de Janeiro, RJ

Claudia Maria Rodrigues Alves

Escola Paulista de Medicina/

Universidade Federal de São Paulo

São Paulo, SP
Cristiano de Oliveira Cardoso

Instituto de Cardiologia do Rio Grande do Sul/

Fundação Universitária de Cardiologia

Porto Alegre, RS

Décio Salvadori Junior

Hospital Beneficência Portuguesa

São Paulo, SP

\section{Esmeralci Ferreira}

Hospital Prontocor

Rio de Janeiro, RJ

Fábio Sândoli de Brito Júnior

Hospital Israelita Albert Einstein

São Paulo, SP

Felipe de Macedo Coelho

Instituto Dante Pazzanese de Cardiologia

São Paulo, SP

Francisco José Araujo Chamié de Queiroz Hospital dos Servidores do Estado/MS-RJ

Rio de Janeiro, RJ

\section{Gederson Rossato}

Hospital Prontocor

Porto Velho, RO

Gilvan Oliveira Dourado

Santa Casa de Maceió

Maceió, AL

Helio José Castello Junior

Hospital Bandeirantes

São Paulo, SP

Hélio Roque Figueira

Hospital Cardiotrauma

Rio de Janeiro, RJ

Helman Campos Martins

Hospital Santa Paula - Cardiocenter

João Pessoa, PB

\section{Ibraim Pinto}

Instituto Dante Pazzanese de Cardiologia

São Paulo, SP 
João Paulo Zouvi

Sociedade Beneficência e Caridade Lajeado

Lajeado, RS

José Ribamar Costa Junior

Instituto Dante Pazzanese de Cardiologia

São Paulo, SP

\section{José Klauber Roger Carneiro}

Hospital do Coração de Sobral

Sobral, CE

Louis N. Ohe

Instituto Dante Pazzanese de Cardiologia

São Paulo, SP

Luciana V. Armaganijan

Instituto Dante Pazzanese de Cardiologia

São Paulo, SP

\section{Luís Henrique Wolff Gowdak}

Instituto do Coração do Hospital das Clínicas da Faculdade de Medicina da USP

São Paulo, SP

\section{Luiz Alberto Mattos}

Instituto Dante Pazzanese de Cardiologia

São Paulo, SP

\section{Luiz Antonio Gubolino}

INCORPI - Hospital dos Fornecedores de

Cana de Piracicaba

Piracicaba, SP

Incor Rio Preto

São José do Rio Preto, SP

\section{Manuel Nicolas Cano}

Instituto Dante Pazzanese de Cardiologia

São Paulo, SP

\section{Marcia Moura Schmidt}

Instituto de Cardiologia do Rio Grande do Sul/

Fundação Universitária de Cardiologia

Porto Alegre, RS

\section{Marco Aurelio de Magalhães}

Hospital Israelita Albert Einstein

São Paulo, SP

\section{Marco Túlio Villaça Castagna}

Hospital São Francisco de Assis

Belo Horizonte, MG

\section{Marinella Centemero}

Instituto Dante Pazzanese de Cardiologia

São Paulo, SP
Miguel Antonio Neves Rati

Hospital Copa D'Or

Rio de Janeiro, RJ

Pedro Beraldo de Andrade

Irmandade da Santa Casa de Misericórdia de Marília Marília, SP

Hospital do Coração

Londrina, PR

\section{Raul D'Aurea Mora}

Hospital Santa Rita

Maringá, PR

Raul Ivo Rossi Filho

Instituto de Cardiologia do Rio Grande do Sul/

Fundação Universitária de Cardiologia

Porto Alegre, RS

\section{Renato Delascio Lopes}

Duke Clinical Research Institute -

Duke University Medical Center

Durham, NC, Estados Unidos

\section{Roberto Vieira Botelho}

Hospital Santa Catarina

Uberlândia, MG

Rodolfo Staico

Instituto Dante Pazzanese de Cardiologia

São Paulo, SP

\section{Rogério Sarmento-Leite}

Instituto de Cardiologia do Rio Grande do Sul/

Fundação Universitária de Cardiologia

Porto Alegre, RS

\section{Said Assaf Neto}

Instituto Dante Pazzanese de Cardiologia

São Paulo, SP

Samuel Silva da Silva

Hospital João de Freitas

Londrina, PR

Sergio Braga

Instituto Dante Pazzanese de Cardiologia

São Paulo, SP

Valmir Fernandes Fontes

Instituto Dante Pazzanese de Cardiologia

São Paulo, SP

Vinicius Daher Vaz

Hospital do Coração Anis Rassi

Goiânia, GO

Wilson Albino Pimentel Filho

Hospital Beneficência Portuguesa

São Paulo, SP 